\title{
Oxaliplatin before autologous transplantation in combination with high-dose cytarabine and rituximab provides longer disease control than cisplatin or carboplatin in patients with mantle-cell lymphoma: results from the LyMA prospective trial
}

\author{
Benoit Tessoulin ${ }^{1,2}$. David Chiron ${ }^{2}$. Catherine Thieblemont ${ }^{3,4} \cdot$ Lucie Oberic $^{5} \cdot$ Kamal Bouadballah $^{6}$. \\ Emmanuel Gyan ${ }^{7,8}$. Gandhi Damaj ${ }^{9,10}$. Vincent Ribrag ${ }^{11}$. Rémy Gressin ${ }^{12}$. Pierre Feugier ${ }^{13}$. \\ Olivier Casasnovas ${ }^{14}$. Hacène Zerazhi ${ }^{15}$. François Lemonnier ${ }^{16}$. Hervé Maisonneuve ${ }^{17}$. Clementine Joubert $^{18}$. \\ Eric Van Den Neste ${ }^{19}$. Thierry Lamy ${ }^{20} \cdot$ Hervé Tilly $^{21} \cdot$ Anne Moreau $^{22} \cdot$ Olivier Hermine $^{23} \cdot$ Steven Le Gouill ${ }^{1,2}$
}

\begin{abstract}
LyMA trial has demonstrated the benefit of rituximab maintenance after autologous stem cell transplantation (ASCT) in previously untreated mantle-cell lymphoma patients (MCL). Induction consisted of four courses of R-DHAP (rituximab, dexamethasone, high-dose cytarabine, and platinum derivative). The platinum derivative (PD) choice was free: R-DHAcisplatin, R-DHA-carboplatin, or R-DHA-oxaliplatin. We investigated the prognostic impact of each PD. PFS and OS calculated from inclusion and investigated in an intention-to-treat (ITT) $(=298)$ and per-protocol analyses (PP) $(n=227)$. RDHACis, R-DHACa, or R-DHAOx were used at first cycle in 184, 76, and 38 patients, respectively. Overall, 71 patients (59 in the R-DHACis) required a change in PD, mainly because of PD toxicity. In ITT-analysis, PFS in the R-DHACis and R-DHACa groups were similar (4-year PFS of 65\%), while R-DHAOx had a better PFS (4-year PFS of 65\% versus $86.5 \%$, respectively, $\mathrm{HR}=0.44, p=0.02)$. The 4-year OS was $92 \%$ for R-DHAOx versus $75.9 \%$ for R-DHACis/DHACa $(\mathrm{HR}=$ $0.37, p=0.03$ ). Similar results were yielded in the PP analysis. Low MIPI and R-DHAOx were independent favorable prognostic markers for both PFS (HR $=0.44, p=0.035)$ and $\mathrm{OS}(\mathrm{HR}=0.36, p=0.045)$. In vitro and in silico analyses confirmed that oxaliplatin has an anti-MCL cytotoxic effect that differs from that of other PD. R-DHAOx before ASCT provides better outcome in transplantation eligible young MCL patients.
\end{abstract}

\section{Introduction}

The DHAP (dexamethasone, high-dose cytarabine plus cisplatin) regimen, first published in 1988 by Velasquez et al., is a standard of care salvage chemotherapy treatment given before or without autologous stem cell transplantation (ASCT) in Hodgkin's and non-Hodgkin's lymphoma (NHL) since its efficacy was demonstrated in the PARMA study in 1995 [1-4]. DHAP with or without rituximab (RDHAP) is used worldwide in daily practice. The side effects associated with (R)-DHAP are well described and include hematological, neurological, gastrointestinal tract, and renal toxicities. However, the use of (R)-DHAP is not completely straightforward because of the need for hyperhydration to prevent cisplatin-induced nephrotoxicity and thus hospitalization. Nevertheless, even well-conducted hyperhydration does not always prevent renal failure. Cisplatin-induced nephrotoxicity is indeed a major issue because it jeopardizes the use of other antitumor agents and presents a contraindication for ASCT. Other platinum derivatives (PD), such as carboplatin and oxaliplatin, have been developed to improve PD antitumor activity while reducing their side effects. Oxaliplatin is mainly used in polychemotherapy regimens delivered in epithelial and digestive tract cancers [5-7], but also finds application in the gemcitabine/ 
oxaliplatin (GEMOX) regimen for relapsed NHLs, while carboplatin is part of the ifosfamide/carboplatin and etoposide regimen. These last two drugs are sometimes substituted for cisplatin in the DHAP regimen despite a lack of prospective comparisons between these three PDs in NHL and very limited published data about R-DHA-carboplatin (R-DHACa) and R-DHA-oxaliplatin (R-DHAOx) in hematological malignancies overall $[8,9]$.

Mantle-cell lymphoma (MCL) is a mature B-cell malignancy that carries the $\mathrm{t}(11 ; 14)$ translocation. Treatment for young patients typically consists of a high-dose cytarabine-containing immunochemotherapy frontline prior to ASCT [10, 11]. The LyMa trial (ClinicalTrials.govNCT00921414) is an international, prospective, randomized, phase III trial that investigated the use of rituximab maintenance (RM) after ASCT in young patients with newly diagnosed MCL. Prior to ASCT, patients received four courses of R-DHA plus a PD as induction chemotherapy. According to the protocol, local investigators were free to choose between cisplatin (R-DHACis), carboplatin (R-DHACa), or oxaliplatin (R-DHAOx). There was no randomization or stratification regarding the three PDs. To date, there is no published data to prospectively compare these three PDs. In the era of novel targeted drugs and immunotherapies, an "old" question like "what is the best PD to use in DHAP?" will never be addressed in a randomized and prospective trial, although it remains an issue relevant to everyday clinical practice. Therefore, we analyzed data collected as part of the LyMa trial to compare the three regimens (R-DHACis, R-DHACa, and R-DHAOx) in patients with MCL. In addition, anti-MCL cytotoxicity and antitumor tissue specificities of each PDs were investigated and compared using in silico and in vitro analyses.

\section{Methods}

\section{Patient population}

All MCL patients enrolled in the LyMa trial were eligible for this study. The design of the study is described in the original manuscript [12]. In brief, patients with newly diagnosed MCL ( $<66$ years) were treated with four courses of R-DHAP followed by ASCT consolidation. Patients with an insufficient response after four courses of R-DHAP received four courses of R-CHOP before ASCT. After ASCT, patients were randomized (1:1) between RM $\left(375 \mathrm{mg} / \mathrm{m}^{2}\right.$ rituximab intravenously every 2 months for 3 years) or observation. The trial included 299 patients, of whom 240 were randomly assigned to RM $(n=120)$ or observation $(n=120)$. According to protocol, the nature of the PD (cisplatin, carboplatin, or oxaliplatin) in R-DHAP was not predefined, but dosing was imposed as follows: cisplatin (continuous infusion) $100 \mathrm{mg} / \mathrm{m}^{2}$ over $24 \mathrm{~h}$ on day 1 (DHACis group), carboplatin AUC $=5 \mathrm{mg} / \mathrm{ml} / \mathrm{min}$ (target area under the concentration versus time curve using the Calvert formula) (DHACa group), and oxaliplatin 130 (recommended dosing) or $100 \mathrm{mg} / \mathrm{m}^{2}$ on day 1 (DHAOx group). Local investigators were free to switch PD during induction. Randomization was not stratified regarding the nature of the PD.

\section{Study objectives}

The objective of the present ancillary analysis was to investigate the role of the PD (cisplatin, carboplatin, and oxaliplatin) given in combination with R-DHA as induction regimen prior to ASCT in untreated MCL patients enrolled in the LyMa trial. We investigated PFS, OS, response rate, and toxicity. All these data were monitored and the database used for the present analysis is the same as in the original analysis.

\section{Statistical analysis}

Time-to-event survival curves were estimated using the Kaplan-Meier method. Time-to-event endpoints in the different groups were compared with log-rank tests and Cox proportional-hazard regression analysis. Patients who withdrew (i.e., all patients who did not undergo randomization for any reason), patients who were lost to follow-up (i.e., all patients who underwent randomization and for whom an outcome was not updated for $>1$ year at the time of the final analysis), and patients who did not have an event, as defined in the protocol at the time of the final analysis, had their data censored at the time of their last visit. Response rates were expressed in percentages with 95\% exact confidence intervals that were based on the Clopper-Pearson method. All statistical analyses were performed using the SAS software, version 9.3 (SAS Institute). The present analysis included all patients for whom the nature of the PD used at cycle 1 was reported in the database. Two patient datasets were defined. In the intention-to-treat PD set (ITT-P set), patient outcome was investigated according to the PD used in the first cycle. The per-protocol PD set (PP-P set) includes patients who received the same PD for the whole duration of induction. For both sets, patients were classified into three groups (socalled R-DHACis, R- DHACa, or R-DHAOx), according to the PD used. A multivariate analysis on survival endpoints taking into account randomization arm (RM or observation) and MIPI groups was planned.

\section{In silico and in vitro analyses}

In silico analyses were carried out from CellMinerCDB databases using a growth inhibition 50 index with Spearman 
Table 1 Patient characteristics at inclusion according to treatment at induction (ITT-P set).

\begin{tabular}{|c|c|c|c|c|c|}
\hline Characteristics & $\begin{array}{l}\text { Total included } \\
\text { patients }(n=298)\end{array}$ & $\begin{array}{l}\text { R-DHACis } \\
(n=184)\end{array}$ & $\begin{array}{l}\text { R-DHACa } \\
(n=76)\end{array}$ & $\begin{array}{l}\text { R-DHAOx } \\
(n=38)\end{array}$ & $p$ value \\
\hline \multicolumn{6}{|l|}{ Age } \\
\hline Median (range) & $57(27-65)$ & $57(29-65)$ & $57(41-65)$ & $56.5(27-65)$ & 0.82 \\
\hline \multicolumn{6}{|l|}{ Sex } \\
\hline Male $(n)$ & $236(79)$ & $150(81)$ & $57(75)$ & $29(76)$ & 0.45 \\
\hline MIPI & & & & & 0.6 \\
\hline Low $(n, \%)$ & $159(53)$ & $99(54)$ & 37 (49) & $23(61)$ & \\
\hline Inter $(n, \%)$ & $82(27.5)$ & $53(29)$ & $22(29)$ & $7(18)$ & \\
\hline $\operatorname{High}(n, \%)$ & $57(19)$ & $32(17)$ & $17(22)$ & $8(21)$ & \\
\hline Ann Arbor stage & 1 & 0 & 0 & 0 & \\
\hline $\mathrm{III} / \mathrm{IV}(n, \%)$ & $279(94)$ & $171(93)$ & $71(93)$ & $36(95)$ & 0.31 \\
\hline \multicolumn{6}{|l|}{$\% \mathrm{Ki}-67$} \\
\hline Missing & 82 & 43 & 27 & 12 & \\
\hline$>30 \%(n)$ & $76(35)$ & $54(38)$ & $15(31)$ & $7(27)$ & 0.4 \\
\hline Morphological variant & & & & & 0.04 \\
\hline Missing & 1 & 0 & 0 & 1 & \\
\hline Blastoid/Pleiomorphic $(n, \%)$ & $35(12)$ & $20(11)$ & $14(18)$ & $1(3)$ & \\
\hline \multicolumn{6}{|l|}{ Completed four cycles of R-DHA with the same PD from cycle 1} \\
\hline No $(n, \%)$ & $71(24)$ & $69(38)$ & $1(1)$ & $1(3)$ & $5 e-14$ \\
\hline \multicolumn{6}{|l|}{ Change of PD after cycle 1} \\
\hline To cisplatin & 1 & NA & 1 & 0 & \\
\hline To carboplatin & 30 & 29 & NA & 1 & \\
\hline To oxaliplatin & 40 & 40 & 0 & NA & \\
\hline \multicolumn{6}{|l|}{$\mathrm{R}-\mathrm{CHOP}$ prior to ASCT } \\
\hline Yes $(n, \%)$ & $20(7)$ & $10(5)$ & $9(12)$ & $1(3)$ & 0.096 \\
\hline Disease status (Cheson 99 criteria) after induction (missing) & 25 & 20 & 3 & 2 & \\
\hline $\mathrm{CR}+\mathrm{CRu}(n, \%)$ & $240(88)$ & $146(89)$ & $59(81)$ & $35(97)$ & \\
\hline $\mathrm{CR}(n, \%)$ & $129(47)$ & $83(51)$ & $26(36)$ & $20(55)$ & 0.06 \\
\hline $\mathrm{CRu}(n, \%)$ & $111(41)$ & $63(38)$ & $33(45)$ & $15(42)$ & \\
\hline $\mathrm{PR}(n, \%)$ & $22(8)$ & $13(8)$ & $8(11)$ & $1(3)$ & \\
\hline $\mathrm{SD}(n, \%)$ & $8(3)$ & $2(1)$ & $6(8)$ & 0 & \\
\hline $\mathrm{PD}(n, \%)$ & $3(1)$ & $3(2)$ & 0 & 0 & \\
\hline \multicolumn{6}{|l|}{ Performed ASCT } \\
\hline Yes $(n, \%)$ & $257(86)$ & $155(84)$ & $65(85)$ & $37(97)$ & 0.08 \\
\hline \multicolumn{6}{|l|}{ Randomization $\operatorname{arm}(n, \%)$} \\
\hline Not randomized & $58(19)$ & $39(21)$ & $17(22)$ & $2(5)$ & 0.04 \\
\hline Rituximab maintenance & 120 & $74(40)$ & $30(39)$ & $16(42)$ & 0.5 \\
\hline Observation & 120 & $71(38)$ & $29(38)$ & $20(53)$ & \\
\hline Disease status at last contact & & & & & 0.19 \\
\hline Alive in CR without prog./relapse & $189(63)$ & $112(61)$ & $46(61)$ & $31(82)$ & \\
\hline Alive after prog. or relapse & $32(11)$ & $21(11)$ & $9(12)$ & $2(5)$ & \\
\hline Dead & 77 (26) & $51(28)$ & $21(27)$ & $5(13)$ & \\
\hline
\end{tabular}

MIPI International Prognostic Index, $C R$ complete response, $C R u$ unconfirmed complete response, $P R$ partial response, $S D$ stable disease, $P D$ progressive disease, ASCT autologous stem cell transplantation.

rank correlation statistics. Cell death was assessed by annexin $\mathrm{V}$ staining after $72 \mathrm{~h}$ exposure to PD dilutions in five MCL cell lines [13], with four independent assays, and the lethal dose 50 (LD50) was subsequently calculated. A Friedman's test was used to compare means of LD50 among the three PDs, and a post-hoc analysis with a two- 
Fig. 1 Consort diagram of platinum derivative usage. In green boxes, each arm represents the first assignment of Platinum Derivative (PD): Carboplatin (Carbo), Oxaliplatin (OxaliP) or Cisplatin (CisP). In case of PD change along treatment course (Blue Boxes), switch is indicated (White Boxes). ITT-P-Set: Intention-To-TreatPlatinum-Set, PP-P-Set: PerProtocol-Platinum-Set.

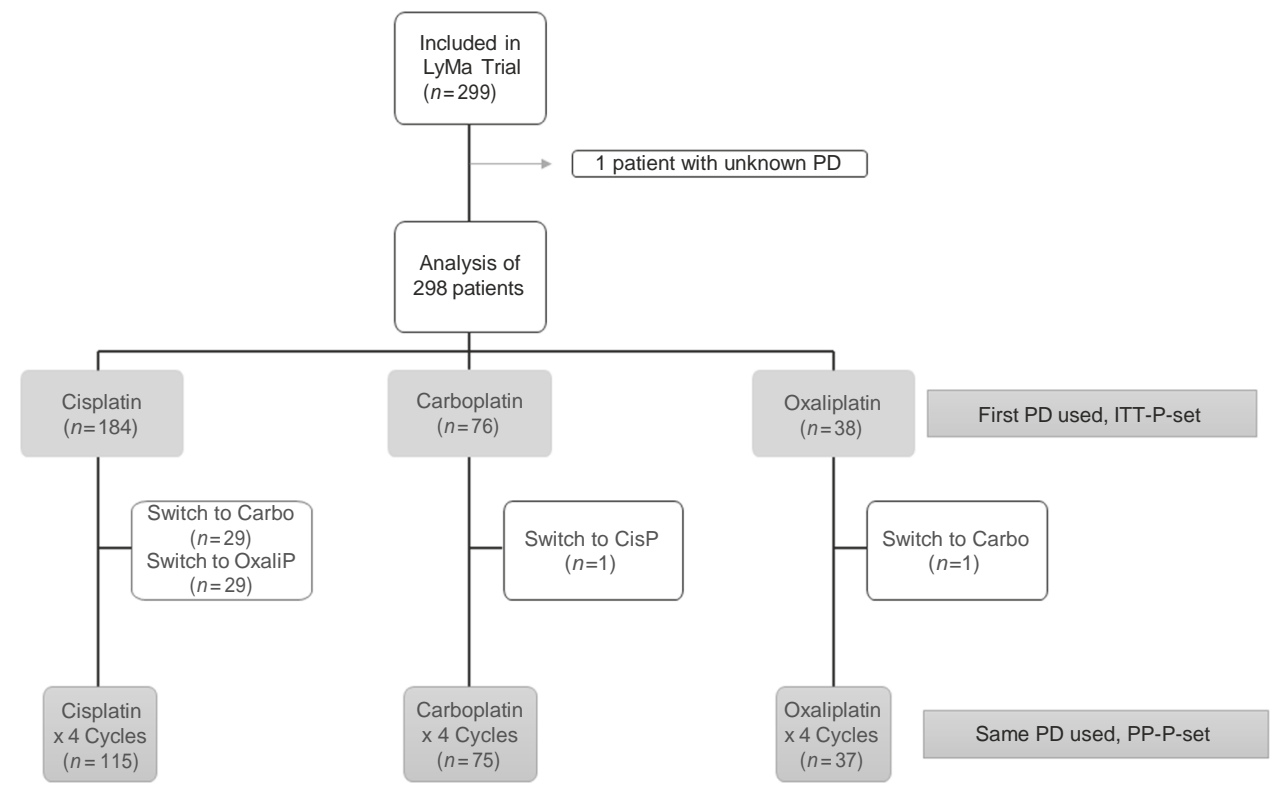

stage linear step-up procedure of Benjamini, Krieger, and Yekutieli false discovery rate was used to compare groups.

\section{Results}

\section{Patient characteristics at inclusion}

Patient characteristics at inclusion according to PD used during the first cycle are presented in Table 1. The characteristics were similar between the three groups, except for the blastic MCL variant that was only found once in the RDHAOx group $(p=0.04)$. Cisplatin was the most frequently used PD at the first cycle $(n=184 ; 61.5 \%)$. Overall, 76 patients $(25.4 \%)$ received R-DHACa and $38(12.7 \%)$ received R-DHAOx. In all, 257 (86\%) patients underwent ASCT, including $155(84 \%)$ patients in the R-DHACis group, $65(85 \%)$ in the R-DHACa group, and $37(97 \%)$ in the R-DHAOx group $(p=0.08)$. In the ITT set, the percentage of R-DHAOx patients who underwent ASCT (97\%) was significantly higher than in the groups receiving $\mathrm{R}$ DHACis or R-DHACa $(85 \%, p=0.04)$. There were more RDHAOx (95\%) patients who were randomized, as compared to R-DHACis (79\%) and R-DHACa (78\%) patients $(p=0.04)$. Finally, an unbalance between blastoid variants was noted among groups $(p=0.04)$ with a reduced proportion in R-DHAOx group. Seventy-one patients did not receive the same $\mathrm{PD}$ during all four courses of R-DHAP. Overall, 69 out of these $71(97 \%)$ patients were in the RDHACis group. Overall, $31(44 \%)$ patients switched at cycle $2,16(22 \%)$ at cycle 3 , and $24(34 \%)$ at cycle 3 (Fig. 1). The choice of the second PD was oxaliplatin in 40 patients $(56 \%)$ and carboplatin in 30 cases $(42 \%)$; only one patient switched from carboplatin to cisplatin.

\section{Toxicities during induction}

We first investigated the relative drug intensity (RDI) for cytarabine, rituximab, and dexamethasone given during induction (Table 2). For aracytine, no differences were noted regarding aracytine RDI between both the ITT $(>90 \%$ of the planned dosing, $p=1)$ and PP (>90\% of the planned dosing, $p=0.88$ ) sets of PD. No differences were noted regarding rituximab RDI between both the ITT $(>90 \%$ of the planned dosing, $p=0.5)$ and PP $(>90 \%$ of the planned dosing, $p=0.1$ ) sets of PD.

Grade 3 and 4 toxicities during induction according to PD group are reported in Table 3. There was no statistical significance in terms of toxicity between the three regimens, but there was a trend for an increased rate of renal dysfunction in the R-DHACis group $(p=0.13)$. Only RDHACis patients had grade 4 renal insufficiency $(n=5)$. The main reasons for not receiving ASCT in the R-DHACis group were insufficient response rate $(n=10)$, renal toxicity $(n=8)$, death $(n=4)$, and other $(n=6$ : ineligible for the procedure, no stem cells, and refusal). The main reasons for not receiving ASCT in the R-DHACa group were insufficient response rate $(n=9)$ and toxicities $(n=2)$. One patient in the R-DHAOx group did not undergo ASCT because of poor performance status before ASCT. More patients in the R-DHACis and R-DHACa groups experienced hematological grade 3 and 4 toxicities (47\% and $42 \%$, respectively) as compared to patients in the $\mathrm{R}$ DHAOx group $(14 \%, p=0.002)$. 


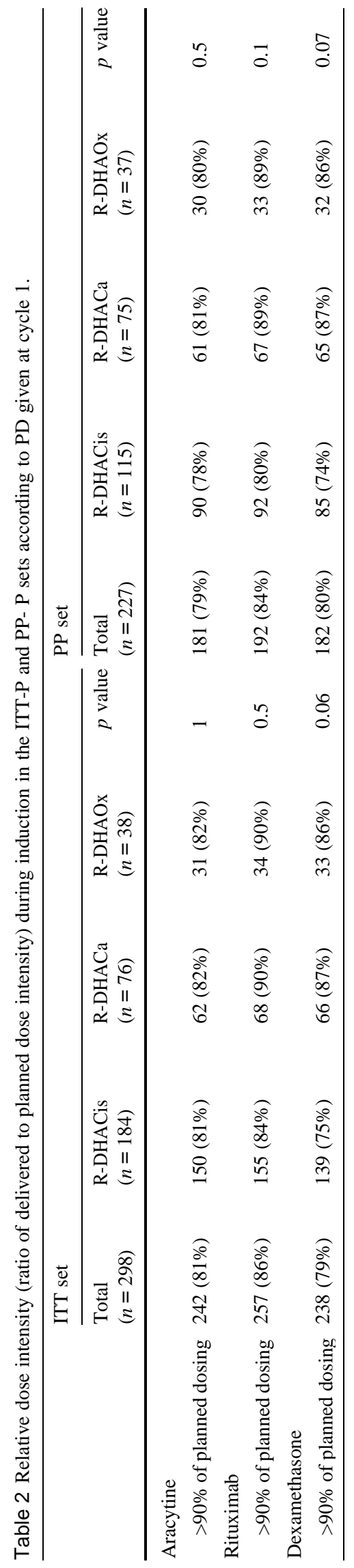

\section{Response and survival}

In the ITT population $(n=298)$, overall response rates after four cycles of induction according to the Cheson 99 criteria were $97 \%$ (complete response/unconfirmed complete response $[\mathrm{CR} / \mathrm{Cru}]$ 89\%) in the R-DHACis group, 92\% (CR/CRu $81 \%$ ) in the R-DHACa group, and $100 \%$ (CR/ $\mathrm{CRu} 97 \%$ ) in the R-DHAOx group. Ten (5\%) patients in the R-DHACis, nine (12\%) in the R-DHACa, and one (3\%) in the R-DHAOx groups did not reach a sufficient response and received four courses of R-CHOP $(p=0.09)$ following R-DHAP.

PFS and OS were calculated from the time of inclusion. There was a trend for better PFS $(p=0.06)$ and OS $(p=0.08)$ in favor of R-DHAOx versus R-DHACis and RDHACa (Fig. 2a, b). The 4-year PFS in the R-DHAOx group was $86.5 \%$ versus $66 \%$ in the R-DHCis and $62 \%$ in the R-DHACa groups $(p=0.06)$. The 4-year OS was $92 \%$ in the R-DHAOx versus $77 \%$ in the R-DHACis and $73 \%$ in the R-DHACa groups $(p=0.08)$. When the RDHACis and R-DHACa groups were combined into one single group and then compared to R-DHAOx, PFS ( $\mathrm{HR}=0.44, p=0.02,95 \% \mathrm{CI}=0.2-0.9)$ was statistically superior in the R-DHAOx group. Overall survival was also superior for R-DHAOx $(\mathrm{HR}=0.37, p=0.03$, 95\% CI $=0.14-1$ ) (Fig. 2c, d). Similar OS and PFS results were obtained in the PP analysis (Fig. 2e-h) and when blastic patients were excluded from both the ITT and PP analyses $(p=0.02$ [ITT] and $p=0.02$ [PP] for PFS; $p=0.03$ [ITT] and $p=0.03$ [PP] for OS). Considering the unbalance regarding "blastoid" status among groups, a dedicated subset analysis was performed: PFS for both ITT-P and PP-P sets were still in favor of RDHAOx $(\mathrm{HR}=0.42, p=0.02$ and $\mathrm{HR}=0.42, p=$ 0.02 , respectively). Similar results were yielded for OS $(\mathrm{HR}=0.33, p=0.03$ for ITT-P and HR $=0.33, p=0.03$ for PP-P set).

The multivariate analysis for both PFS (HR $=0.437$, $p=0.035,95 \% \mathrm{CI}=0.2-0.94)$ and $\mathrm{OS}(\mathrm{HR}=0.36, p=$ $0.045,95 \% \mathrm{CI}=0.13-0.97)$ confirmed R-DHAOx as an independent prognostic variable (Table 4). Considering the number of patients by group, the multivariate analysis could not take into account the RM (well balanced in both ITT-P ( $p=0.8)$ and PP-P $(p=0.7)$ sets). Patients who switched from one PD to another during induction $(n=71)$ had similar PFS and OS as the other patients $(n=227)(p=0.3$ and $p=0.25$, respectively, Fig. $2 \mathrm{i}, \mathrm{j}$ ). Regarding the primary endpoint of the LyMa trial, i.e., event-free survival (EFS) calculated from the time of randomization (after induction plus ASCT), there was no difference according to PD (4-year EFS $=74 \%$ for R-DHAOx and $69 \%$ for R-DHACis $/ \mathrm{Ca}$; HR $=0.61,95 \% \mathrm{CI}=0.28-$ $1.3, p=0.61)$. 
Table 3 Grade 3 and 4 toxicities during induction according to treatment at induction in both ITT-P and PP-P sets.

\begin{tabular}{lcclll}
\hline & $\begin{array}{l}\text { Total } \\
(n=298)\end{array}$ & $\begin{array}{l}\text { R-DHACis } \\
(n=184)\end{array}$ & $\begin{array}{l}\text { R-DHACa } \\
(n=76)\end{array}$ & $\begin{array}{l}\text { R-DHAOx } \\
(n=38)\end{array}$ & $p$ value \\
\hline Mucositis & $3(1)$ & $2(1)$ & 0 & $1(2.6)$ & 0.47 \\
Digestive tract & $20(6.7)$ & $18(10)$ & 0 & $2(5)$ & 0.005 \\
Neurological toxicities & $6(2)$ & $4(2.2)$ & $1(1.3)$ & $1(2.6)$ & 1 \\
Elevated bilirubin & $1(0.3)$ & $1(0.5)$ & 0 & 0 & 1 \\
Creatinine elevation & $15(5)$ & $13(7)$ & $1(1)$ & $1(2)$ & 0.13 \\
\hline
\end{tabular}

\section{In silico and in vitro analyses}

In order to investigate the anti-MCL cytotoxic effects of each PD used alone, we performed in silico and in vitro analyses. The in silico analyses showed that carboplatin, cisplatin, and oxaliplatin display a different sensitivity among cell lines and tissues (see Fig. 3). The GI50 values for carboplatin and cisplatin were strongly correlated $(r=0.83, p=3 \mathrm{e}-16)$, while there was no correlation between cisplatin $(r=0.17$, $p=0.23)$ or carboplatin $(r=0.12, p=0.4)$ and oxaliplatin. In contrast, the GI50 value for oxaliplatin was highly correlated to other PDs, such as tetraplatin $(r=0.8, p=1 \mathrm{e}-11)$ and carboxyphthalatoplatinum $(r=0.8, p=5 \mathrm{e}-12)$.

For the in vitro analyses, we treated five MCL cell lines with various doses of oxaliplatin, carboplatin, or cisplatin. Induced cell death was measured using annexin V staining (see Fig. 4). Cisplatin and oxaliplatin displayed similar toxicity ranges, with a mean LD50 of $0.29(95 \% \mathrm{CI}=$ $0.03-0.55$ ) versus 0.45 (95\% CI $=0.01-0.9)$, respectively, $(p=0.26)$. In contrast, carboplatin $(1.7,95 \%$ CI $=0.07-$ 3.3) appeared less cytotoxic than cisplatin $(p=0.012)$ and oxaliplatin $(p=0.014)$.

\section{Discussion}

Our results show that R-DHACis, R-DHACa, and RDHAOx are not equal in terms of anti-MCL toxicity, which might influence patient outcomes. One limitation of the present study is the lack of randomization or stratification between the three induction regimens, but all data were monitored, and patients were treated in a large phase III prospective trial. A consequence of this limitation is the rather low number of patients treated by R-DHAOx in this trial. Furthermore, missing data may have undermined some results, especially regarding Ki-67 staining. Although when analyzing missing data pattern, the missing data were randomly distributed among PD groups $(p=0.11)$.

Published data about DHAOx and DHACa are scarce. Tessoulin et al. show in a monocentric retrospective study that DHACa (with or without R) can be safely used in various lymphoma entities, including MCL. DHACa was shown to have similar efficacy and a better toxicity profile as compared to DHACis [8]. Lignon et al. reported a monocentric study of R-DHAOx (oxaliplatin was used at the dose of $100 \mathrm{mg} / \mathrm{m}^{2}$ ) [14] and Tixier et al. conducted a retrospective multicenter study and compared the 3 PDs [9]. These last two reports demonstrate the higher risk of nephrotoxicity, renal failure, and auditory toxicities with DHACis, while patients treated with DHAOx are more at risk of moderate neurotoxicity. Our results are in line with those of previous reports. Indeed, 69 out of 71 patients who switched PD were in the R-DHACis group $(37.5 \%$ of the patients of the R-DHACis group, $p=1 \mathrm{e}-14)$. This reflects the serious toxicity profile of R-DHACis. The reason to change PD during induction was not always recorded in the database, but toxicity, in particular renal dysfunction, was frequently reported for these patients. Accordingly, 8 out of 41 patients that did not undergo ASCT had renal insufficiency and all had been exposed to cisplatin. It is also important to notice that R-DHAOx and R-DHACa are more convenient to use in daily practice than R-DHACis. Both regimens do not require intravenous hyperhydration and can be delivered in an outpatient unit. In summary, both for safety and ease of administration, R-DHAOx and RDHACa are more convenient than R-DHACis.

In terms of disease control, our work shows that there is no difference between R-DHACa and R-DHACis, while there is a trend in favor of R-DHAOx. The reason to explain these discrepancies between the three regimen is the choice of PD during induction. Indeed, drugs intensity deliveries for dexamethasone, cytarabine, and rituximab were the same in the three regimens. The nature of PD did also not influence patients' outcome calculated from the time of randomization (after ASCT), which suggests that the gain in PFS and OS in favor of R-DHAOx occurred during induction. A better outcome for patients in the R-DHAOx group was not only reported in the ITT set, but also in the PP analysis. In the latter group, patients could receive the full planned course of induction with the same PD. The better result with RDHAOx in the PP analysis indeed suggests that oxaliplatin may possess superior anti-MCL cytotoxicity compared to the two other PDs and that the benefit for PFS and OS in the R-DHAOx group may not only be due to its better tolerability. Both the in silico and in vitro analyses confirm this finding. The three PDs display different mechanisms of action. The anti-MCL activity of carboplatin appears inferior compared to that of cisplatin and oxaliplatin. These last two 
a

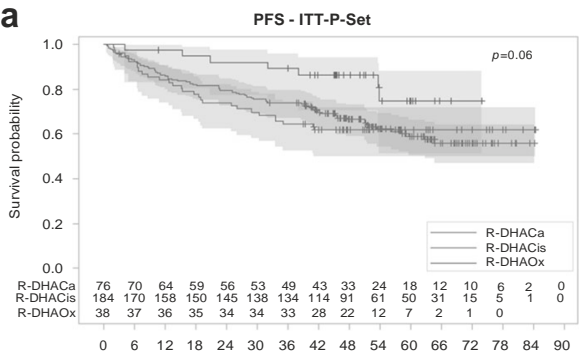

b

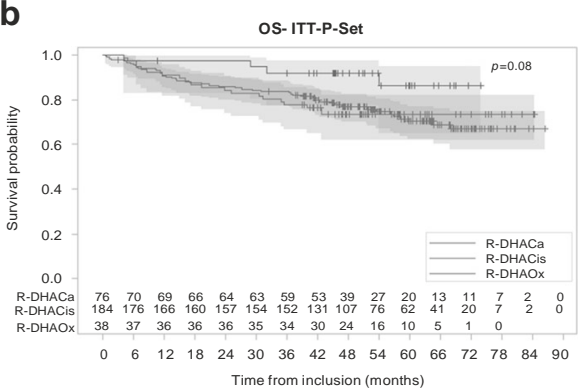

e

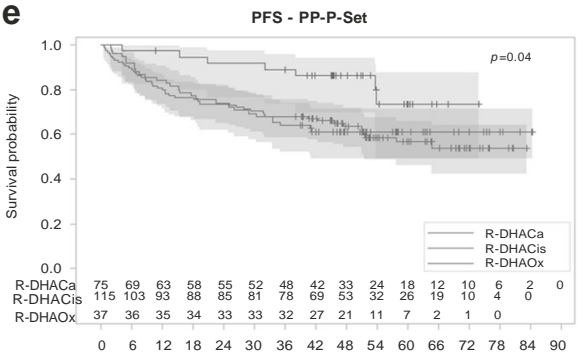

f

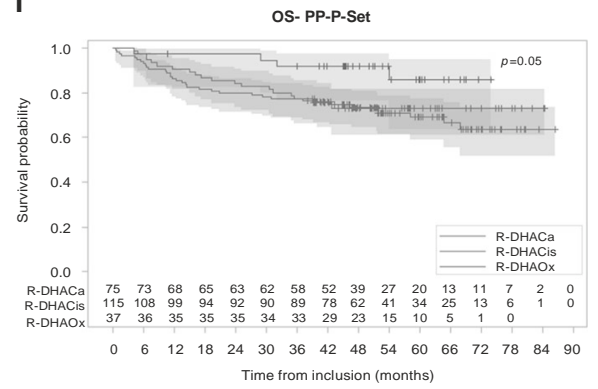

i

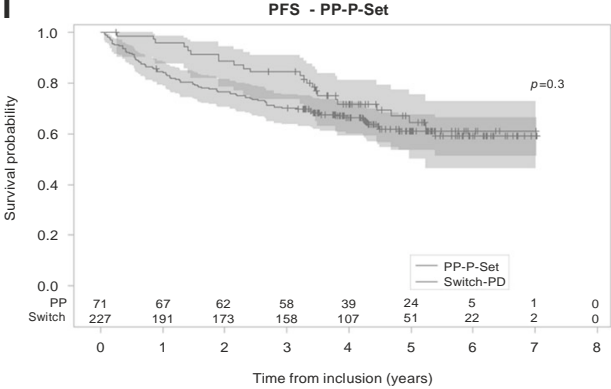

Fig. 2 Kaplan-Meier estimates of progression free survival (PFS) and overall survival (OS). a PFS in the ITT-P set according to induction regimen. $\mathrm{b}$ OS in the ITT-P set according to induction regimen. c PFS in the ITT-P set comparing R-DHAOx versus RDHACis plus R-DHACa. $d$ OS in the ITT-P set comparing R-DHAOx versus R-DHACis plus R-DHACa. e PFS in the PP-P set according to induction regimen. $\mathrm{f}$ OS in the PP-P set according to induction
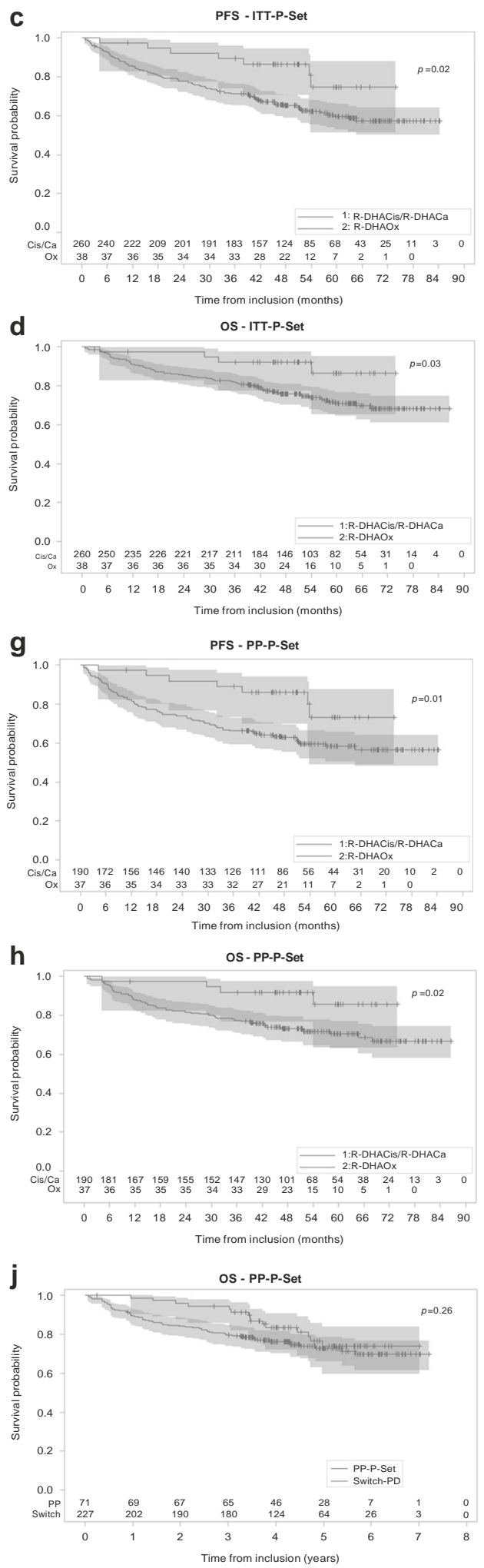

regimen. $g$ PFS in the PP-P set comparing R-DHAOx versus RDHACis plus R-DHACa. h OS in the PP-P set comparing R-DHAOx versus R-DHACis plus R-DHACa. i PFS for patients who switched of PD during induction versus PP set patients. $\mathrm{j}$ OS for patients who switched of PD during induction versus PP set patients. $p$ values are derived from the likelihood-ratio chi-squared test of the considered model. 
Table 4 Multivariate analysis of PFS and OS in the ITT-P set.

\begin{tabular}{|c|c|c|c|c|c|c|}
\hline \multirow[b]{2}{*}{ Variable } & \multicolumn{3}{|l|}{ PFS } & \multirow{2}{*}{$\begin{array}{l}\mathrm{OS} \\
\mathrm{HR}\end{array}$} & \multirow[b]{2}{*}{$p$ value } & \multirow[b]{2}{*}{$95 \% \mathrm{CI}$} \\
\hline & HR & $p$ value & $95 \% \mathrm{CI}$ & & & \\
\hline Oxaliplatin & 0.437 & 0.0351 & $0.203-0.944$ & 0.355 & 0.0446 & $0.129-0.975$ \\
\hline MIPI-int. & 1.702 & 0.0241 & $1.072-2.70$ & 1.827 & 0.0415 & $1.024-3.26$ \\
\hline MIPI-high & 3.064 & $<0.0001$ & $1.929-4.87$ & 4.188 & $<0.0001$ & $2.422-7.24$ \\
\hline
\end{tabular}

MIPI-Int. intermediate risk according to the International Prognostic Index, MIPI-High high risk according to the International Prognostic Index.
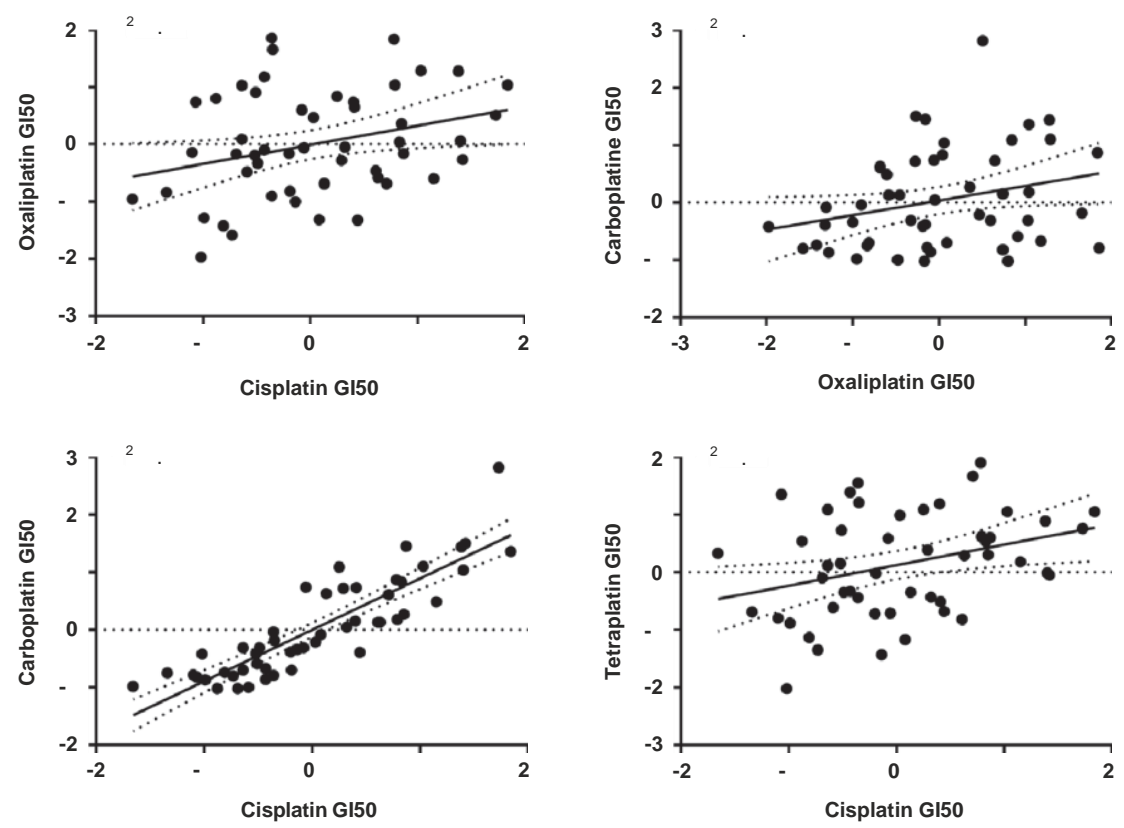

Fig. 3 Linear regression analysis of growth index 50 (extracted from CellMinerCDB, $n=50$ cell lines with complete data for four PDs, GI50 values are centered and scaled). The solid line represents

the linear regression line of the considered PDs, dashed lines represent the $95 \%$ CI. The $r^{2}$ value is provided.

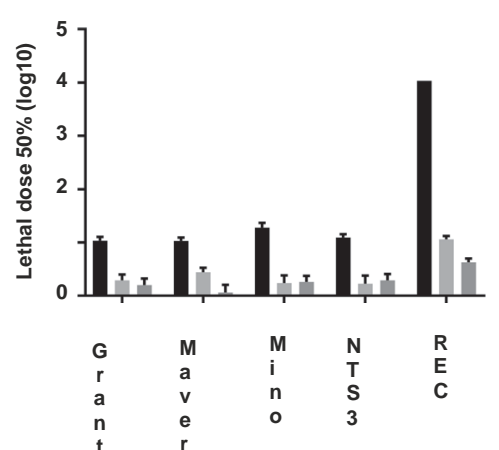

Fig. 4 Platinum derivative-i thduced $^{r}$ cell death measured by annexin $\mathrm{V}$ staining in five MCL cell lines (REC, Mino, MAVER, GRANTA, and NTS3). MCL cells were treated in standard condition at various

drugs display similar apoptosis-induced cell death rates. In silico investigations suggest that there is a tissue-specific profile of efficacy for each PD, which cannot be detected by in vitro experiments. Some authors have reported that oxaliplatin has little to low cross-resistance with cisplatin [15]. A Spanish group reported both in vivo and in vitro results in

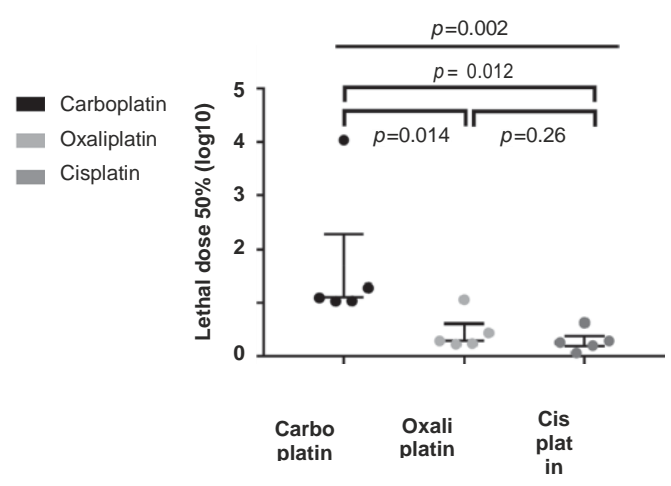

doses of oxaliplatin, carboplatin, and cisplatin as indicated. Left panel: Lethal dose 50 in five MCL cell lines, mean with standard error of the mean (SEM). Right panel: Scatterplots of LD50, with 95\% CI.

line with ours [16]. A combination of rituximab and GEMOX was delivered to 30 patients (10 diagnosis and 20 with R/R MCLs). The ORR was $83 \%$, with $60 \%$ of complete response, furthermore in vitro data demonstrated that oxaliplatin (contrary to cisplatin) had a synergistic effect when combined with cytarabine. Hence, the differences between 
the three PDs suggest that the role of PDs in disease control in MCL should not be underestimated similar to other hematological malignancies. The importance of the PDs might explain the unexpected negative result reported in the MCL-5 trial conducted by the Nordic Lymphoma Group, in which young patients with newly diagnosed MCL were treated with high-dose cytarabine without dexamethasone and PD before ASCT. The trial prematurely stopped because of the unexpected high incidence of relapse [17]. The absence of a PD during induction may explain the lack of efficacy of this study.

In conclusion, R-DHAOx and R-DHACa have a better safety profile than R-DHACis and R-DHAOx provides longer disease control in young treatment-naive MCL patients who are eligible for ASCT. Our result supports the use of R-DHAOx during induction in MCL.

\section{References}

1. Gisselbrecht C, Glass B, Mounier N, Singh Gill D, Linch DC, Trneny M, et al. Salvage regimens with autologous transplantation for relapsed large B-cell lymphoma in the rituximab era. J Clin Oncol. 2010;28:4184-90.

2. Philip T, Chauvin F, Armitage J, Bron D, Hagenbeek A, Biron P, et al. Parma international protocol: pilot study of DHAP followed by involved- field radiotherapy and BEAC with autologous bone marrow transplantation. Blood. 1991;77:1587-92.

3. Philip T, Guglielmi C, Hagenbeek A, Somers R, Van Der Lelie H, Bron D, et al. Autologous bone marrow transplantation as compared with salvage chemotherapy in relapses of chemotherapy-sensitive non-Hodgkin's lymphoma. N Engl J Med. 1995;333:1540-5.

4. Velasquez WS, Cabanillas F, Salvador P, McLaughlin P, Fridrik $\mathrm{M}$, Tucker S, et al. Effective salvage therapy for lymphoma with cisplatin in combination with high-dose Ara-C and dexamethasone (DHAP). Blood. 1988;71:117-22.

5. Rothenberg ML, Oza AM, Bigelow RH, Berlin JD, Marshall JL, Ramanathan RK, et al. Superiority of oxaliplatin and fluorouracilleucovorin compared with either therapy alone in patients with progressive colorectal cancer after irinotecan and fluorouracilleucovorin: interim results of a phase III trial. J Clin Oncol. 2003;21:2059-69.

6. Hironaka S, Sugimoto N, Yamaguchi K, Moriwaki T, Komatsu Y, Nishina T, et al. S-1 plus leucovorin versus S-1 plus leucovorin and oxaliplatin versus S-1 plus cisplatin in patients with advanced gastric cancer: a randomised, multicentre, open-label, phase 2 trial. Lancet Oncol. 2016;17:99-108.

7. Mehmood RK. Review of cisplatin and oxaliplatin in current immunogenic and monoclonal antibody treatments. Oncol Rev. 2014;8. https://www.ncbi.nlm.nih.gov/pmc/articles/PMC4419649/.

8. Tessoulin B, Thomare P, Delande E, Moynard J, Gastinne T, Moreau A, et al. Carboplatin instead of cisplatin in combination with dexamethasone, high-dose cytarabine with or without rituximab (DHAC+/-R) is an effective treatment with low toxicity in Hodgkin's and non-Hodgkin's lymphomas. Ann Hematol. 2017;96:943-50.

9. Tixier F, Ranchon F, Iltis A, Vantard N, Schwiertz V, Bachy E, et al. Comparative toxicities of 3 platinum-containing chemotherapy regimens in relapsed/refractory lymphoma patients. Hematol Oncol. 2017;35:584-90.

10. Dreyling M, Campo E, Hermine O, Jerkeman M, Le Gouill S, Rule S, et al. Newly diagnosed and relapsed mantle cell lymphoma: ESMO Clinical Practice Guidelines for diagnosis, treatment and follow-up. Ann Oncol. 2017;28:iv62-71.

11. Hermine O, Hoster E, Walewski J, Bosly A, Stilgenbauer S, Thieblemont $\mathrm{C}$, et al. Addition of high-dose cytarabine to immunochemotherapy before autologous stem-cell transplantation in patients aged 65 years or younger with mantle cell lymphoma (MCL Younger): a randomised, open-label, phase 3 trial of the European Mantle Cell Lymphoma Network. Lancet. 2016;388:565-75.

12. Le Gouill S, Thieblemont C, Oberic L, Moreau A, Bouabdallah K, Dartigeas $\mathrm{C}$, et al. Rituximab after autologous stem-cell transplantation in mantle-cell lymphoma. $\mathrm{N}$ Engl $\mathrm{J}$ Med. 2017;377:1250-60.

13. Papin A, Tessoulin B, Bellanger C, Moreau A, Le Bris Y, Maisonneuve $\mathrm{H}$, et al. CSF1R and BTK inhibitions as novel strategies to disrupt the dialog between mantle cell lymphoma and macrophages. Leukemia. 2019;33:2442-53.

14. Lignon J, Sibon D, Madelaine I, Brice P, Franchi P, Briere J, et al. Rituximab, dexamethasone, cytarabine, and oxaliplatin (RDHAX) is an effective and safe salvage regimen in relapsed/ refractory B-cell non-Hodgkin lymphoma. Clin Lymphoma Myeloma Leuk. 2010;10:262-9.

15. Rixe O, Ortuzar W, Alvarez M, Parker R, Reed E, Paull K, et al. Oxaliplatin, tetraplatin, cisplatin, and carboplatin: spectrum of activity in drug-resistant cell lines and in the cell lines of the National Cancer Institute's Anticancer Drug Screen panel. Biochem Pharmacol. 1996;52:1855-65.

16. Obrador-Hevia A, Serra-Sitjar M, Rodríguez J, Belayachi L, Bento L, García-Recio M, et al. Efficacy of the GemOx-R regimen leads to the identification of oxaliplatin as a highly effective drug against mantle cell lymphoma. Br J Haematol. 2016;174:899-910.

17. Laurell A, Kolstad A, Jerkeman M, Räty R, Geisler CH. High dose cytarabine with rituximab is not enough in first-line treatment of mantle cell lymphoma with high proliferation: early closure of the Nordic Lymphoma Group mantle cell lymphoma 5 trial. Leuk Lymphoma. 2014;55:1206-8. 